\title{
Density of phonon states in cubic ice Ic
}

\author{
Leonardo del Rosso, ${ }^{*, \dagger}$ Milva Celli, ${ }^{\dagger}$ Daniele Colognesi, ${ }^{\dagger}$ Svemir Rudić, ${ }^{\dagger}$ Niall J. \\ English, ${ }^{\mathbb{T}}$ and Lorenzo Ulivi ${ }^{\dagger}$
}

Consiglio Nazionale delle Ricerche, Istituto di Fisica Applicata "Nello Carrara", via Madonna del Piano 10, I-50019 Sesto Fiorentino, Italy, ISIS Facility, STFC Rutherford Appleton Laboratory, Chilton, Oxfordshire OX11 OQX, United Kingdom, and School of Chemical and Bioprocess Engineering, University College Dublin, Belfield, Dublin 4, Ireland

E-mail: I.delrosso@ifac.cnr.it

June 11, 2021

\begin{abstract}
The measurement of the H-projected density of phonon states (H-DOPS) of polycrystalline ice Ic has been performed with an unprecedented accuracy, and in a sample having an almost perfect crystallographic purity, as it was obtained from the transformation of ice XVII. Results are compared with new accurate measurements of H-DOPS in ice Ih, and with centroid molecular-dynamics (MD) computations. The differences between the experimental H-DOPS in these two forms of ice are subtle, but quantitatively measurable. In addition, they are reproduced semi-quantitatively by computational methods, demonstrating the effectiveness of this innovative simulation tool for reproducing the dynamical properties of the ice structures.
\end{abstract}

\footnotetext{
*To whom correspondence should be addressed

†Consiglio Nazionale delle Ricerche, Istituto di Fisica Applicata "Nello Carrara", via Madonna del Piano 10, I-50019 Sesto Fiorentino, Italy

$\ddagger$ ISIS Facility, STFC Rutherford Appleton Laboratory, Chilton, Oxfordshire OX11 0QX, United Kingdom

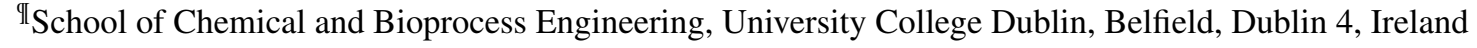




\section{Introduction}

The investigation of microscopic properties of ice has always attracted an extreme interest of researchers in many different science fields. Aside its fundamental role in biological systems, ice represents a paradigmatic case of study for material science because this simple molecular crystal gives rise to a complex phenomenology, among which the ice "anomalous" properties and polymorphism are only two of the most significant aspects. ${ }^{1}$ Indeed, since the beginning of the $20^{\text {th }}$ century with the Tamman's and Bridgman's studies, ${ }^{2,3}$ a lot of experimental and computational efforts are being made to characterize, both from structural and dynamical points of view, the different crystalline phases that water ice can assume, ${ }^{4}$ also in order to better understand the role played by the hydrogen bond in the anomalous properties of liquid water. ${ }^{5}$ To date, 19 different polymorphs of ice have been experimentally discovered and structurally determined, even if several more have been computationally predicted. ${ }^{6-9}$ At ambient pressure, hexagonal ice Ih is the stable form of ice, but, for a long time, the existence of a further polytype of ice I, i.e. the cubic ice Ic, represented a long debated issue in the water ice research area. ${ }^{10}$ Its presence in nature, especially in the atmosphere, was inferred by various experimental observations, ${ }^{11}$ among which the Scheiner's halo is the most suggestive, ${ }^{12}$ but the low difference in free energy between the two polytypes ${ }^{13}$ hinders the possibility to easily access this phase. As a matter of fact, a lot of attempts were made in the past to synthesize pure ice Ic, ${ }^{14,15}$ but all the claimed cubic ice I forms were essentially stacking-disordered forms of ice I, named ice Isd, with larger or lower "cubicity", i.e. proportion of cubic stacking sequences present in the sample, determined by the route followed in the sample synthesis. ${ }^{14,16}$ Only recently two independent experimental studies have revealed new routes to obtain structurally pure cubic ice, either by heating an ice XVII sample, ${ }^{17}$ or by evacuating a $\mathrm{C}_{2}$ hydrogen hydrate, ${ }^{18}$ together with another recent study demonstrating the effectiveness of the heterogeneous nucleation for the ice Ic synthesis. ${ }^{19}$

The microscopic dynamics of water ice, in all of its structural forms, either crystalline or amorphous, is of great importance and has been deeply investigated. 1,20-24 The differences in the phonon density of states among several phases of ice can be revealed by comparing inelastic 
neutron scattering data measured on high-resolution spectrometers, which enable to record scattering events with an exchanged wave vector $Q \neq 0$, not accessible by the standard photon scattering techniques, e.g. IR and Raman. ${ }^{25}$ Referring to the common hexagonal ice Ih, easily obtained by freezing liquid water at ambient pressure, since 1969 few experimental studies have been devoted to determine accurate phonon dispersion curve on fully deuterated single crystal, ${ }^{20,26}$ while a correct assignment of the measured phonon spectral features to the different phonon branches, also supported by lattice dynamics calculations, was performed by Li et al. ${ }^{21}$ On the contrary, as for the more elusive cubic structure of ice I, the experimental outcomes presented in the same paper ${ }^{21}$ cannot be fully assigned to a pure cubic structure, but rather to a stacking-disordered form of ice I, as the analysed sample was obtained by warming recovered ice II and VI samples. ${ }^{14}$ Useful indications about the phonon dynamics can be obtained from density functional theory (DFT) sim-

ulations regarding the proton-ordered form of ice Ic, ${ }^{27}$ while, for its proton-disordered counterpart, reliable computational results are still missing.

In this paper we will present the results of a combined experimental and computational study on the H-DOPS of $\mathrm{H}_{2} \mathrm{O}$ cubic ice. The same kind of investigation on the hexagonal phase Ih, obtained by the transformation of the mentioned cubic ice sample, has allowed us to quantitatively measure and successfully interpret the differences in the phonon spectra of the two polytypes, further confirmed by centroid molecular dynamics (MD) simulations.

\section{Methods}

\section{Sample preparation}

The $\mathrm{H}_{2} \mathrm{O}$ cubic ice sample for the present INS experiment was obtained starting from a hydrogen filled ice in the $\mathrm{C}_{0}$ phase, i.e. a $\mathrm{H}_{2}-\mathrm{H}_{2} \mathrm{O}$ binary compound synthesized in a powder form at high pressure (above $4 \mathrm{kbar}$ ) and at temperature $T=250 \mathrm{~K}$, that can be recovered at ambient pressure by quenching it at $77 \mathrm{~K}$. Further details about the high-pressure synthesis procedure, performed at IFAC-CNR (Italy), are reported in Ref. [29]. The sample was then transferred in an optical vacuum 
tight cell, inserted in a cryostat and equipped with a gas handling system, that allowed us, first by means of Raman scattering, to check the quality of the starting sample and second, to apply the necessary annealing treatment so as to obtain an empty ice XVII. ${ }^{30,31}$ The recovered ice XVII sample was then shipped to the ISIS facility (RAL, STFC, U.K.) in a Dewar cooled by a liquid nitrogen bath. The ice XVII powder was then transferred in a standard flat aluminium cell (1 mm thickness) equipped with two temperature sensors placed at the opposite sides of the cell body to ensure a good thermal control and placed into the TOSCA cryostat through a standard center stick. All the handling procedures of the recovered samples, both $\mathrm{C}_{0}$ filled ice and ice XVII, were always performed in a dry nitrogen atmosphere keeping a good thermal contact with a liquid nitrogen bath to preserve the sample phase.

\section{Experiment}

The measurements have been performed on TOSCA, ${ }^{32}$ a neutron spectrometer devoted to the study of the vibrational dynamics through the Inelastic Neutron Scattering (INS) technique. TOSCA is a crystal-analyzer inverse-geometry spectrometer with final energy, $E_{1}$, fixed at about $3.3 \mathrm{meV}$, selected via two sets of pyrolytic graphite crystals placed at two selected scattering angles. Highorder Bragg reflections are eliminated through a beryllium filter kept at low temperature. The excellent energy resolution $\left(\Delta \hbar \omega / E_{0} \simeq 1.5 \% \div 3 \%\right)$ and the wide region of accessible energy transfer ( $\hbar \omega=E_{0}-E_{1}$, from 3 to $500 \mathrm{meV}$, where $E_{0}$ is the incident energy) make the TOSCA spectrometer particularly useful to study the vibrational dynamics of the polycrystalline ice sample. All the neutron measurements reported in this work have been performed at $T=20 \mathrm{~K}$. The collected time-of-flight data ${ }^{33}$ were automatically transformed into energy transfer spectra by a dedicated routine present in the MANTID software. ${ }^{34}$ Owing to the fully hydrogenated nature of the sample, a $3 \mathrm{~h}$ acquisition time was sufficient to collect an INS spectrum with a extremely small statistical error, crucial to pinpoint differences between the ice Ic and Ih. 


\section{Simulation}

We took a $3 \times 3 \times 3$ supercell of ice $\mathrm{Ic}^{17}$ as well as $5 \times 3 \times 3$ of ice $\mathrm{Ih}$ from Hayward-Reimers ${ }^{35}$ and simulated these two samples at $170 \mathrm{~K}$ and $50 \mathrm{~K}$, with lattice constants $a=6.37451$ And $a=6.36434 \AA$ A , respectively. ${ }^{17}$ We carried out centroid dynamics with 64 beads in the i-PI software package ${ }^{36}$ using the many body water potential MB-pol ${ }^{37}$ as part of the MBX software package, ${ }^{38}$ in conjunction with Nosé-Hoover thermostat, ${ }^{39,40}$ Ewald summation, ${ }^{41}$ and a time-step of $0.1 \mathrm{fs}$ for $100 \mathrm{ps}$, including $10 \mathrm{ps}$ for system equilibration.

The MB-pol interaction model, used together with centroid MD, has been noted to perform very well for simulating ice thermodynamic and dynamical properties. ${ }^{42-44}$ We obtained the lattice phonon DOS from the Fourier cosine transformation of the averaged velocity autocorrelation function of the oxygen atoms. ${ }^{41}$ In addition, the phonon dispersion curves were also extracted from the MD data. ${ }^{45}$

\section{Results and discussion}

Preliminarly, the neutron spectrum of the recovered $\mathrm{H}_{2} \mathrm{O}$ ice XVII sample was measured at $T=20$ $\mathrm{K}$ for about $1 \mathrm{~h}$ (integrated proton current, IPC=168.4 $\mu \mathrm{Ah}$ ), verifying that no deterioration of the ice XVII sample during its shipping to ISIS, the filling of the TOSCA cell, and its insertion in the cryostat has occured. After this check, the sample was heated to $150 \mathrm{~K}$ and kept at this temperature for about $1 \mathrm{~h}$, so to induce the transition from ice XVII to ice Ic. Then the sample was cooled again to $20 \mathrm{~K}$ and the spectrum of ice Ic was measured for about $3 \mathrm{~h}$ (IPC=583.6 $\mu \mathrm{Ah}$ ). Afterward, the sample was heated at $268 \mathrm{~K}$ and maintained at this temperature for about $1 \mathrm{~h}$ to induce the transition from ice Ic to ice Ih. Finally, the sample was cooled down to $20 \mathrm{~K}$ and the spectra of ice Ih was measured for about $10 \mathrm{~h}$ (IPC=1811.1 $\mu \mathrm{Ah}$ ). The analysis of the experimental INS data on ice Ic and Ih was done in a way similar to what has been presented in Ref. [46]. The first two steps consisted in the subtraction of the empty-cell scattering contributions, and the correction for the well-known self-shielding attenuation, both performed through the analytical approach proposed 
by Sears. ${ }^{47}$ Then multiple scattering contributions made of two (or more) inelastic scattering events have been approximately estimated and found rather small in the $\hbar \omega$ interval of interest (namely, below $130 \mathrm{meV}$ ). For this reasons no multiple scattering corrections were operated on the two INS spectra. Finally, in order to calculate and subtract the multiphonon terms in our data, we set up a self-consistent procedure ${ }^{48}$ already successfully used on a number of ice samples. ${ }^{49}$ Such an approach, which fully exploits the incoherent approximation, ${ }^{50}$ was completely justified for a polycrystalline material formed by $\mathrm{H}_{2} \mathrm{O}$, leading almost directly to the desired $\mathrm{H}$-DOPS. This is due to the much larger neutron cross section, and lower mass, of the proton than that of the oxygen nucleus.

In spite of the approximate character of the multiphonon self-consistent procedure, which assumes a purely incoherent, harmonic, and isotropic treatment of the multiphonon contributions, its practical implementation showed a rapid convergence of the method, and made possible a reliable extraction of the one-phonon component of the self scattering law, ${ }^{50}$ from which the H-DOPS, $Z_{\mathrm{H}}(\omega)$, of both Ic and Ih could be extracted up to $\hbar \omega=130 \mathrm{meV}$, that is the region where the librational bands end. Bending and stretching bands of $\mathrm{H}_{2} \mathrm{O}$ have been clearly left out of the analysis since the neutron spectrometer used was not optimized for their accurate detection as the $Q$ values corresponding to their typical $\hbar \omega$ ranges were by far too high.

It is worth noticing that a very accurate result would require the subtraction of the tiny $\mathrm{O}$ contributions from the experimental estimates of $Z_{\mathrm{H}}(\omega)$. The magnitude of these contributions was estimated with the help of the MD simulations and found to be totally negligible in the librational part of the phonon spectra, while quite small, but still visible, in the lattice one. However, since lattice phonons always correspond to collective motions of the $\mathrm{H}_{2} \mathrm{O}$ molecules seen as single rigid units, then the $\mathrm{H}$ and $\mathrm{O}$ contributions to the density of phonon states in this spectral zone show exactly the same spectral shape. For this reason, the $O$ scattering corrections to $Z_{\mathrm{H}}(\omega)$ could be rigorously performed by scaling the overall intensity of the lattice part of $Z_{\mathrm{H}}(\omega)$ (i.e., that in the $\hbar \omega$ range from 0 to $50 \mathrm{meV}$ ) by an appropriate factor, and then normalizing the corrected $Z_{\mathrm{H}}(\omega)$ to the requested area. 
The extracted $Z_{\mathrm{H}}(\omega)$ for the starting phase of ice XVII, and both cubic and hexagonal ice I, are reported in Figure 1.

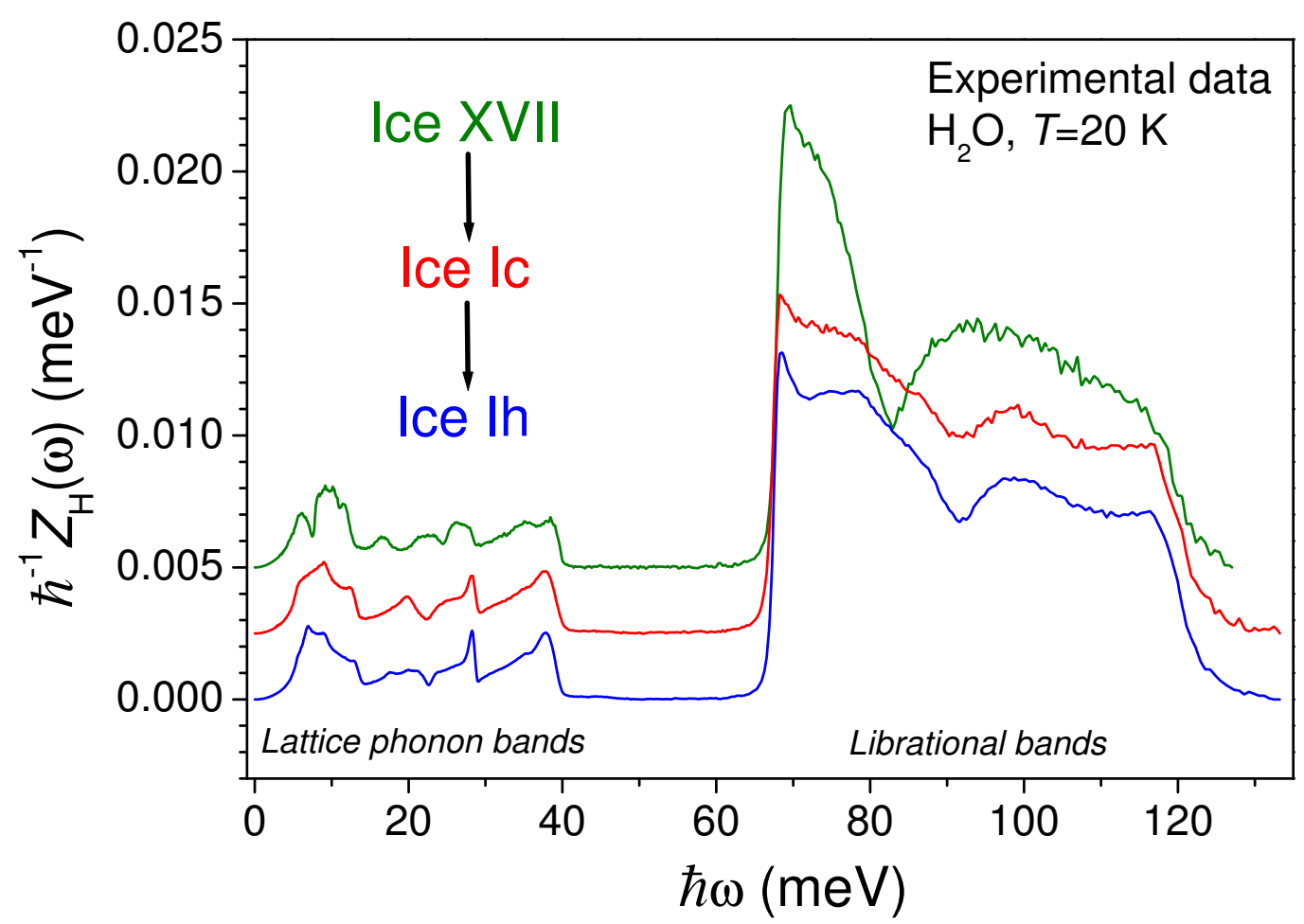

Figure 1: Hydrogen-projected density of phonon states of the ice XVII (green line), ice Ic (red line), and ice Ih (blue line) extracted from INS data collected at $20 \mathrm{~K}$ on TOSCA neutron spectrometer. To ease the comparison, the red and green curves are vertically shifted of $0.0025 \mathrm{meV}^{-} 1$ and $0.0050 \mathrm{meV}^{-} 1$, respectively.

From this plot we can easily recognize two well-separated groups of bands relative to the rigid $\mathrm{H}_{2} \mathrm{O}$ molecule vibrations, i.e. the lattice phonon modes, both acoustic and optic $(\hbar \omega<45 \mathrm{meV})$, and the librational modes, i.e. wagging, rocking and twisting ${ }^{27}(65 \mathrm{meV}<\hbar \omega<130 \mathrm{meV})$. By looking at the former type of bands of both ice I structures, one can observe that the lattice phonon region of $Z_{\mathrm{H}}(\omega)$ is quite similar to what can be observed in other ice forms, even at high pressure, ${ }^{21}$ but is different from the lattice bands pertaining to empty hydrate structures, like ice $\mathrm{XVI}^{51}$ and ice XVII, ${ }^{46,52}$ that show a cleft of the main acoustic peak at about $7 \mathrm{meV}$. Also the librational bands show a quite different shape passing from the open and channelled structure of the ice XVII to the cubic and hexagonal structure of ice I, with a more intense principal peak at 
about $70 \mathrm{meV}$ and a deeper groove placed at $80 \mathrm{meV}$ in the ice XVII. This spectral change, quite common in the ice landscape, e.g. the open structure of the ice II, ${ }^{21}$ is explainable by the different environment experienced by the water molecule when so different ice structures are considered. In addition, by comparing the measured spectra of the cubic and hexagonal ice, it appears quite clear that the acoustic band is the most sensitive to the different arrangement of layers present in the two structures. Such marked differences were not reported by Li et al., ${ }^{21}$ probably because of the lower cubicity of their sample. Moreover, the substantially identical onset of the acoustic band reflects the same long-range order that the $\mathrm{H}_{2} \mathrm{O}$ molecules experience in both structures. On the other hand, in the optic band no change of the shape is recorded passing from ice Ic to ice Ih, but rather a different intensity ratio of the peaks located at $27 \mathrm{meV}$ and $38 \mathrm{meV}$, in a way similar to what has been measured by Raman spectroscopy on the lattice band $\left(200-320 \mathrm{~cm}^{-1}\right)$ during the Ic-Ih phase transformation. ${ }^{28}$ Small differences can be also found in the librational region of $Z_{\mathrm{H}}(\omega)$, in particular for the shallower and less pronounced dip at about $92 \mathrm{meV}$.

Through centroid MD simulations, we have calculated the density of phonon states, which is compared in Figure 2 with the same quantity extracted from INS spectra. On average, the comparison with the computed $Z_{\mathrm{H}}(\omega)$ shows a surprising good quality, similar to what was obtained for clathrate hydrates of various structures, ${ }^{53,54}$ and the simulated curves show the same type of shape change passing from the cubic to the hexagonal structure.

Furthermore, in Figure 3 the simulated phonon dispersion curve has been plotted side by side with the experimental H-DOPS for both ice Ih and Ic in the energy region of the lattice phonon modes. Most of the peaks in the H-DOPS curves have a clear correspondence in energy with the flat (i.e. zero derivative) regions of the dispersion curves. So from this comparison, in the case of ice Ic, it is possible to clearly assign a purely acoustic and optic character to the first spectral band centred around $10 \mathrm{meV}$ and the last centred around $37 \mathrm{meV}$, respectively. However, as for the intermediate bands at $19 \mathrm{meV}$ and $28 \mathrm{meV}$, one observes that they originate from the partial overlapping of different phonon branches, with the former having mainly an acoustic character, while the latter a substantial optic character. It is worth noting that this kind of assignment is in 


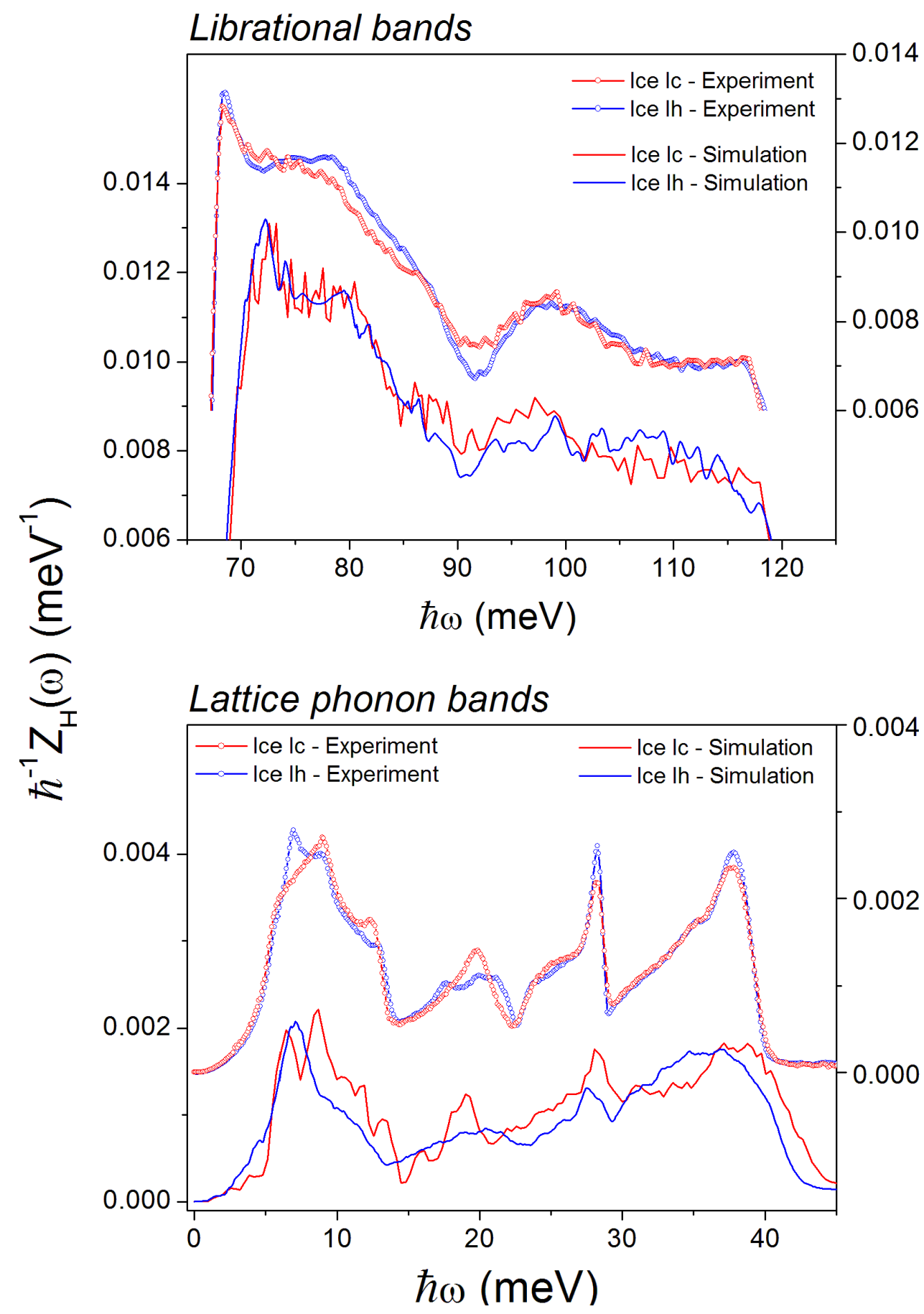

Figure 2: Comparison of the hydrogen-projected density of phonon states belonging to ice Ic and ice Ih (lattice phonon bands, lower panel, and librational bands, upper panel) extracted from INS data collected at $20 \mathrm{~K}$ on TOSCA neutron spectrometer, and from centroid MD simulations performed at $50 \mathrm{~K}$. 
accordance with what has been observed for the ice Ih case. ${ }^{21,57}$
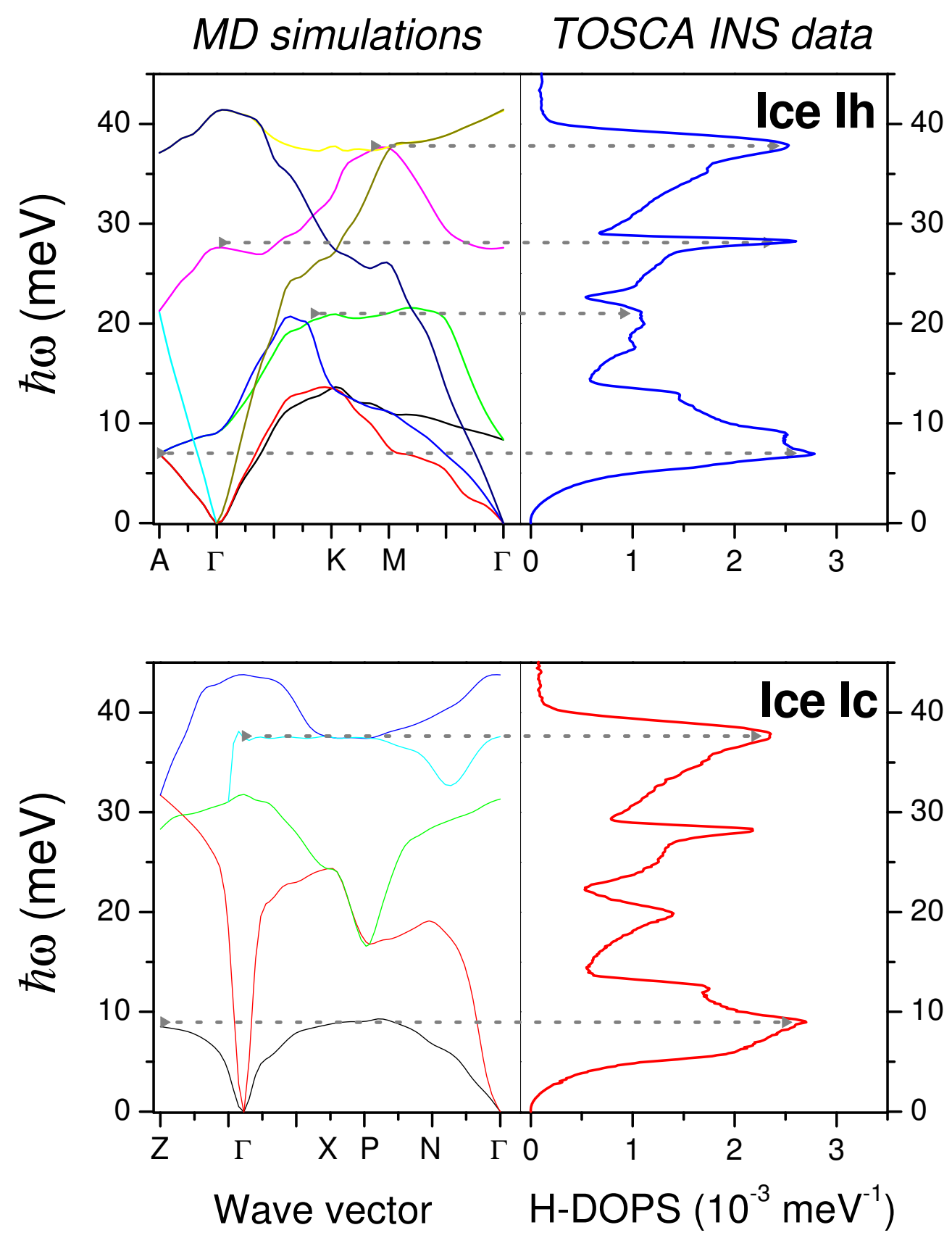

Figure 3: MD simulated phonon dispersion curve at $50 \mathrm{~K}$ (left plots) and experimental H-DOPS extracted by TOSCA INS data at $20 \mathrm{~K}$ (right plots) for the two ice I polytypes (hexagonal structure, upper panel; cubic structure, lower panel). Both right plots share the same energy axis and the dotted lines are only a guide for the eye. 
The good performance of MB-pol and centroid MD in Figures 2 and 3 is rather striking, although this general approach has shown to be very robust in performance related to the ice vibrational properties. ${ }^{42-44}$ Indeed, it seems that the quality of this highly accurate polarisable water model, in tandem with quantum dynamics, is needed to truly capture the intricacies of ice polytypes with near-quantitative accuracy. This appears to emerge from the rich interplay of quantum effects at low temperatures together with with the need for highly accurate electronic-structuretrained wave-functions ${ }^{37}$ under the conditions of condensed-matter crystal symmetries. The two Ih and Ic optic modes at 28 and $37 \mathrm{meV}$ are reproduced to some extent, as well as the $19 \mathrm{meV}$ Ic one. In general, for these peaks classical MD based on standard pairwise potentials is less good than polarisable potentials, and even sophisticated Thole-type models ${ }^{54}$ are not as good as the MBpol used in the present study. However, it must be borne in mind that quantum dynamics was not employed in the Burnham's study, which compared, inter alia, different popular potential modes for ice Ih. The present centroid-MD/MB-pol predictions are quantitatively superior to various recent DFT prediction of phonon DOS in ice polymorphs, especially ice Ih, which have still shed important and interesting light, nonetheless, on contributions from O-O interactions and hydrogen bonding. ${ }^{55-57}$ However, the present computational results in Figures 2 and 3 are more similar in phonon character for the two polytypes of ice I to experimental than DFT results of Ref.s 22-27, owing to the combined accuracy of MB-pol and also to centroid-MD sampling, with the latter an especially important feature at $50 \mathrm{~K}$. Still, for the relatively minor discrepancies between the experimental and simulation results in Figures 2 and 3, these are explained mainly by MB-pol's slight differences in hydrogen-bonding structure and symmetry, and possibly by the imperfect nature of centroid-MD to capture fully and rigorously dynamical effects. ${ }^{58}$

\section{Conclusions}

The INS measurements reported in this study have led to the determination of the density of phonon states for the cubic structure of ice I. Owing to the accuracy of the measurements on the pure cubic 
phase and on the hexagonal one, which is directly obtained by warming the same sample, we were able to effectively compare the dynamics of these two phases, quantitatively measuring the subtle, but significative, spectral changes before and after the phase transformation. The combined effect of the high resolution of the experimental data and the reliability of the centroid-MD/MBpol computational method have also allowed to perform a clear assignment of the spectral bands for both ice I polytypes, demonstrating the high effectiveness of this innovative computational approach to reproduce the dynamics of the ice systems. This combined study represents so far, to our knowledge, one of the most accurate attempts to computationally investigate phonons in ice, despite the approximate character of the centroid-MD, given the very accurate nature of the MB-pol model. In this perspective, in the future it will be particularly interesting to perform INS

measurements on a single crystal of cubic ice in order to have a direct access to the different phonon dispersion curves, as well as to study the ice Ic dynamics considering a possible proton ordering.

\section{Acknowledgement}

We want to thank Francesco Paesani for tips and help with MBX and i-PI.

\section{References}

(1) Petrenko, V.; Withworth R. Physics of Ice; Oxford University Press: Oxford, U.K., 1999.

(2) Tammann, G. Ueber die Grenzen des festen Zustandes IV. Ann. Phys. 1900, 307, 1-31.

(3) Bridgman, P. W. Water, in the Liquid and Five Solid Forms, under Pressure. Proc. Am. Acad. Arts Sci. 1912, 47, 441-558.

(4) Bartels-Rausch, T., et al. Ice Structures, Patterns, and Processes: A View across the Icefields. Rev. Mod. Phys. 2012, 84, 885-944.

(5) Li, J.; Ross, D. K. Evidence for Two Kinds of Hydrogen Bond in Ice. Nature 1993, 365, 327-329. 
(6) Salzmann, C. G. Advances in the Experimental Exploration of Water's Phase Diagram. $J$. Chem. Phys. 2019, 150, 060901.

(7) Loerting, T.; Fuentes-Landete, V.; Tonauer, C. M.; Gasser, T. M. Open Questions on the Structures of Crystalline Water Ices. Comm. Chem. 2020, 3, 1-4.

(8) Gasser, T. M.; Thoeny, A. V.; Fortes, A. D.; Loerting, T. Structural Characterization of Ice XIX as the Second Polymorph Related to Ice VI. Nat. Commun. 2021, 12, 1-10.

(9) Hansen, T. C. The everlasting hunt for new ice phases. Nat. Commun. 2021, 12, 1-3.

(10) Salzmann, C. G.; Murray, B. J. Ice Goes Fully Cubic. Nat. Mater. 2020, 19, 586-587.

(11) Shilling, J. E.; Tolbert, M. A.; Toon, O. B.; Jensen, E. J.; Murray, B. J.; Bertram, A. K. Measurements of the Vapor Pressure of Cubic Ice and Their Implications for Atmospheric Ice Clouds. Geophys. Res. Lett. 2006, 33, 1-5.

(12) Riikonen, M.; Sillanpaa, M.; Virta, L.; Sullivan, D.; Moilanen, J.; Luukkonen, I. Halo Observations Provide Evidence of Airborne Cubic Ice in the Earth's Atmosphere. Geophys. Res. Lett. 2000, 39, 6080-6085.

(13) Handa, Y. P.; Klug, D. D.; Whalley, E. Difference in Energy between Cubic and Hexagonal Ice. J. Chem. Phys. 1986, 84, 7009-7010.

(14) Malkin, T. L.; Murray, B. J.; Salzmann, C. G.; Molinero, V.; Pickering, S. J.; Whale, T. F. Stacking Disorder in Ice I. Phys. Chem. Chem. Phys. 2015, 17, 60-76.

(15) Amaya, A. J. et al. How Cubic Can Ice Be? J. Chem. Phys. Lett. 2017, 8, 3216-3222.

(16) Kuhs, W. F.; Sippel, C.; Falenty, A.; Hansen, T. C. Extent and Relevance of Stacking Disorder in Ice Ic. Proc. Natl. Acad. Sci. U.S.A. 2012, 109, 21259-21264.

(17) del Rosso, L.; Celli, M.; Grazzi, F.; Catti, M.; Hansen, T. C.; Fortes, A. D.; Ulivi, L. Cubic Ice Ic without Stacking Defects Obtained from Ice XVII. Nat. Mater. 2020, 19, 663-668. 
(18) Komatsu, K.; Machida, S.; Noritake, F.; Hattori, T.; Sano-Furukawa, A.; Yamane, R.; Yamashita, K.; Kagi, H. Ice Ic without Stacking Disorder by Evacuating Hydrogen from Hydrogen Hydrate. Nat. Commun. 2020, 11, 464.

(19) Davies, M. B.; Fitzner, M.; Michaelides, A. Routes to Cubic Ice through Heterogeneous Nucleation. Proc. Natl. Acad. Sci. U.S.A. 2021, 118, e2025245118.

(20) Renker, B. Physics and Chemistry of Ice; University of Toronto Press: Toronto, U.S.A, 1973.

(21) Li, J. Inelastic Neutron Scattering Studies of Hydrogen Bonding in Ices. J. Chem. Phys. 1996, $105,6733-6755$.

(22) Bennington, S. M.; Li, J.; Harris, M. J.; Ross, D. K. Phonon Softening in Ice Ih. Phys. B: Cond. Matt. 1999, 263, 396-399.

(23) Strassle, T.; Saitta, A. M.; Klotz, S.; Braden, M. Phonon Dispersion of Ice Under Pressure. Phys. Rev. Lett. 2004, 93, 225901.

(24) Peng, Z.; Yang, L.; Hui, Y.; Sheng-Hao, H.; Ying-Bo, L.; Mao-Shui, L.; Wei-Yan, C. New Observations on Hydrogen Bonding in Ice by Density Functional Theory Simulations. Chin. Phys. B 2014, 23, 026103.

(25) Wong, P. T. T.; Whalley, E. Optical Spectra of Orientationally Disordered Crystals. VI. The Raman Spectrum of the Translational Lattice Vibrations of Ice Ih. J. Chem Phys. 1976, 65, 829-836.

(26) Renker, B. Phonon Dispersion in $\mathrm{D}_{2} \mathrm{O}-$ Ice. Phys. Lett. 1969, 30A, 493-494.

(27) Yuan, Z. Y.; Zhang, P.; Yao, S. K.; Lu, Y. B.; Yang, H. Z.; Luo, H. W.; Zhao, Z. J. Computational Assignments of Lattice Vibrations of Ice Ic. $R S C A d v . \mathbf{2 0 1 7}, 7,36801-36806$.

(28) Celli, M.; Ulivi, L.; del Rosso, L. Raman Investigation of the Ice Ic-Ice Ih Transformation. J. Phys. Chem. C 2020, 124, 17135-17140. 
(29) del Rosso, L.; Celli, M.; Ulivi, L. A New Porous Water Ice Stable at Atmospheric Pressure Obtained by Emptying a Hydrogen Filled Ice. Nat. Commun. 2016, 7, 13394/1-13394/7.

(30) del Rosso, L.; Celli, M.; Colognesi, D.; Grazzi, F.; Ulivi, L. Irreversible Structural Changes of Recovered Hydrogen Hydrate Transforming from $\mathrm{C}_{0}$ Phase to Ice XVII. Chem. Phys. 2021, 7, 111092.

(31) del Rosso, L.; Grazzi, F.; Celli, M.; Colognesi, D.; Garcia-Sakai, V.; Ulivi, L. Refined Structure of Metastable Ice XVII from Neutron Diffraction Measurements. J. Phys. Chem. C 2016, $120,26955-26959$.

(32) Colognesi, D.; Celli, M.; Cilloco, F.; Newport, R. J.; Parker, S. F.; Rossi-Albertini, V.; Sacchetti, F.; Tomkinson, J.; Zoppi, M. TOSCA Neutron Spectrometer: The Final Configuration. Appl. Phys. A 2002, 74, s64-s66.

(33) Ulivi, L.; Rudić, S.; Celli, M.; del Rosso, L.; Colognesi, D. Lattice Dynamics of Ice XVII Filled with Monatomic and Diatomic Gases. STFC ISIS Neutron and Muon Source 2019.

(34) Taylor, J.; et al. Mantid, A High Performance Framework for Reduction and Analysis of Neutron Scattering Data. Bulletin of the American Physical Society 2012, 57, 1.

(35) Hayward, J. A.; Reimers, J. R. Unit Cells for the Simulation of Hexagonal Ice. J. Chem. Phys. 1997, 106, 1518-1529.

(36) Kapil, V.; et al. i-PI 2.0: A Universal Force Engine for Advanced Molecular Simulations. Comp. Phys. Comm. 2019, 236, 214-223.

(37) Medders, G. R.; Babin, V.; Paesani, F. Development of a "First-Principles" Water Potential with Flexible Monomers. III. Liquid Phase Properties. J. Chem. Theory Comput. 2014, 10, 2906-2910.

(38) The Paesani Research Group: MBX. http://paesanigroup.ucsd.edu/software/mbx.html (accessed 2021-05-24). 
(39) Nosé, S. A Unified Formulation of the Constant Temperature Molecular Dynamics Methods. J. Chem. Phys. 1984, 81, 511-519.

(40) Hoover, W. G. Canonical Dynamics: Equilibrium Phase-Space Distributions. Phys. Rev. A 1985, 31, 1695-1697.

(41) Allen, M. P.; Tildesley, D. J. Computer Simulation of Liquids, 2nd Ed.; Oxford University Press: Oxford, U.K., 2017.

(42) Pham, C. H.; Reddy, S. K.; Chen, K.; Knight, C.; Paesani, F. Many-Body Interactions in Ice. J. Chem. Theory Comput. 2017, 13, 1778-1784.

(43) Moberg, D. R.; Straight, S. C.; Knight, C.; Paesani, F. Molecular Origin of the Vibrational Structure of Ice Ih. J. Phys. Chem. Lett. 2017, 8, 2579-2583.

(44) Moberg, D. R.; Sharp, P. J.; Paesani, F. Molecular-Level Interpretation of Vibrational Spectra of Ordered Ice Phases. J. Phys. Chem. B 2018, 122, 10572-10581.

(45) McGaughey, A. J.; Larkin, J. M. Predicting Phonon Properties from Equilibrium Molecular Dynamics Simulation. Annu. Rev. Heat Transf. 2014, 17, 49-87.

(46) del Rosso, L.; Celli, M.; Colognesi, D.; Rudić, S.; English, N. J.; Burnham, C. J.; Ulivi, L. Dynamics of Hydrogen Guests in Ice XVII Nanopores. Phys. Rev. Materials 2017, 1, 065602.

(47) Sears, V. F. Slow-Neutron Multiple Scattering. Adv. Phys. 1975, 24, 1-45.

(48) Dawidowski, J.; Bermejo, F. J.; Granada, J. R. Efficient Procedure for the Evaluation of Multiple Scattering and Multiphonon Corrections in Inelastic Neutron-Scattering Experiments. Phys. Rev. B 1998, 58, 706-715.

(49) Kolesnikov, A. I.; Li, J.-C. Multiphonon Contributions in Inelastic Neutron Scattering Spectra of Ice. Physica B 1997, 234, 34-36.

(50) Lovesey, S. W. Neutron Scattering, vol. 1; Clarendon Press: Oxford, U.K., 1984. 
(51) Wang, Z. R.; Zhu, X. L.; Jiang, L.; Zhang, K.; Luo, H. W.; Gu, Y.; Zhang, P. Investigations of the Hydrogen Bonds and Vibrational Spectra of Clathrate Ice XVI. Materials 2019, 12, 246.

(52) Zhu, X. L.; Yuan, Z. Y.; Jiang, L.; Zhang, K.; Wang, Z. R.; Luo, H. W.; Luo, H. W.; Gu, Y.; Cao, J. W.; Qin, X. L.; Zhang, P. Computational Analysis of Vibrational Spectrum and Hydrogen Bonds of Ice XVII. New J. Phys. 2019, 21, 043054.

(53) Celli, M.; Colognesi, D.; Ulivi, L.; Zoppi, M.; Ramirez-Cuesta, A. J. Phonon Density of States in Different Clathrate Hydrates Measured by Inelastic Neutron Scattering. J. Phys: Conf. Ser. 2012, 340, 012051.

(54) Burnham, C. J.; English, N. J. Study of Clathrate Hydrates via Equilibrium MolecularDynamics Simulation Employing Polarisable and non-Polarisable, Rigid and Flexible Water Models. J. Chem. Phys. 2016, 144, 164503.

(55) Chen, S.; Xu, Z.; Li, J. The Observation of Oxygen - Oxygen Interactions in Ice. New J. Phys. 2016, $18,023052$.

(56) Tian, L.; Kolesnikov, A. I.; Li, J. Ab Initio Simulation of Hydrogen Bonding in Ices under Ultra-High Pressure. J. Chem. Phys. 2012, 137, 204507.

(57) Zhang, P.; Tian, L.; Zhang, Z. P.; Shao, G.; Li, J. Investigation of the Hydrogen Bonding in Ice Ih by First-Principles Density Function Methods. J. Chem. Phys. 2012, 137, 044504.

(58) Willatt, M. J.; Ceriotti, M.; Althorpe, S. C. Approximating Matsubara Dynamics Using the Planetary Model: Tests on Liquid Water and Ice. J. Chem. Phys. 2018, 148, 102336. 